\title{
The role of the skin barrier in modulating the effects of common skin microbial species on the inflammation, differentiation and proliferation status of epidermal keratinocytes
}

Patrick Duckney ${ }^{1,2}$, Heng Kuan Wong ${ }^{2}$, José Serrano ${ }^{2}$, Diaraf Yaradou ${ }^{2}$, Thierry Oddos ${ }^{2}$ and Georgios N Stamatas ${ }^{3^{*}}$

\begin{abstract}
Background: Skin resident microbial species are often thought of either as pathogenic or commensal. However, little is known about the role of the skin barrier in modulating their potential for causing disease. To investigate this question we measured the effects of three microbial species commonly found on the skin (Staphylococcus epidermidis, Staphylococcus aureus, and Propionibacterium acnes) on a reconstructed human epidermal model by either applying the bacteria on the model surface (intact barrier) or adding them to the culture medium (simulating barrier breach).

Results: When added to the medium, all of the tested species induced inflammatory responses and keratinocyte cell death with species-specific potency. P. acnes and S. epidermidis induced specific alterations in the expression of keratinocyte differentiation and proliferation markers, suggesting a barrier reparation response. S. aureus induced complete keratinocyte cell death. On the contrary, topically applied S. epidermidis and P. acnes caused no inflammatory response even when tested at high concentrations, while topical $S$. aureus induced a weak reaction. None of the tested species were able to alter the expression of keratinocyte differentiation or expression markers, when applied topically.

Conclusions: We show that the skin barrier prevents the effects of common skin bacteria on epidermal keratinocyte inflammation, differentiation and proliferation and highlight the importance of skin barrier in defending against the pathogenic effects of common skin bacteria.
\end{abstract}

Keywords: Microbial infection, Skin barrier, Stratum corneum, Reconstructed human epidermis, Skin microbiome, Keratinocyte differentiation, Inflammation

\section{Background}

In the last few years it has been conclusively demonstrated that the human skin is an important part of the innate immune system, acting as a first barrier against external microbial threats. In response to pathogenic insults, epidermal keratinocytes secrete an array of soluble cytokines and chemokines to initiate an active immune response [1]. A number of innate defensive pathways are activated, including the generation of reactive oxygen bursts $[2,3]$ and the secretion of human $\beta$-defensin, cathelicidin and RNase-family antimicrobial peptides [3].

\footnotetext{
* Correspondence: GStamata@its.jnj.com

${ }^{3}$ Johnson \& Johnson Santé Beauté France, 1 rue Camille Desmoulins, Issy-les-Moulineaux 92787, France

Full list of author information is available at the end of the article
}

It is becoming increasingly accepted that commensal species of micro-organisms that naturally reside on the surface of the human skin are an integral part of the innate immune system. For example, Staphylococcus epidermidis, the most common cutaneous bacteria, secretes antimicrobial phenol-soluble modulins [4], while stimulating secretion of antimicrobial peptides from keratinocytes, providing increased resistance to pathogen infection [5]. Colonization of the skin by the common microflora may assist in training the developing adaptive immune system by providing epitopes against which the immune system can become primed [6,7]. S. epidermidis for example, controls cutaneous immune responses by modulating local T-effector cells through the IL-1 pathway, establishing resistance to infection [8]. The compositions of species 
that colonize the skin differ between skin sites in correlation with the physiological conditions of the skin microenvironment [9]. Furthermore, the composition of the human skin microflora matures following birth and throughout infancy [6] and possibly through puberty, as the human skin matures in terms of sebaceous activity [10], surface water content [11] and skin surface $\mathrm{pH}$ [12]. It could be expected that the skin adjusts its physiological conditions to favor colonization by commensal microflora species over pathogenic species during this period.

However, the same commensal microbial species are also implicated in the pathogenesis of cutaneous infections under altered micro-environmental conditions or when able to overcome the host immune systems [13], as well as in proinflammatory diseases such as acne vulgaris [14], atopic dermatitis [15], and psoriasis [16]. Upon penetration of the skin barrier, members of the skin microflora have been known to elicit diseases such as impetigo, cellulitis, and systemic diseases including endocarditis and sepsis [17]. Knowledge of the immunological interactions between the skin keratinocytes and the skin microflora is imperative in understanding the role of the skin microflora in immune protection and pathogenesis, as well as the factors that determine the commensal or pathogenic nature of the skin microbiome.

During the first years of life, the barrier function and microstructure of infant skin continue to mature $[11,18]$. It is possible that the early colonization of the skin by commensal bacteria and the subsequent maturation of microbial colony compositions [6] may influence the correct development of the epidermis following birth. Microbial-induced effects on keratinocyte differentiation and proliferation have been observed previously: P. acnes has been shown to stimulate normal human epidermal keratinocyte (NHEK) proliferation and affect expression of the differentiation markers Filaggrin, $\beta 1$ Integrin [19], Transglutaminases 1, 3 and 5, and Keratins 1, 10 and 17 [20]. The ability of members of the skin microbiome to modify epidermal keratinocyte properties and the comaturation of infant epidermal properties with microflora species composition over time may suggest that the skin microflora may be important for skin maturation in early life.

The top layer of human skin, the stratum corneum, presents a physical barrier to microbial migration through the skin. To investigate the importance of the skin barrier in modulating the effects of the skin microflora on the human epidermis, we applied three species of common skin colonizers to the Reconstructed Human Epidermis (RHE) model. The model consists of stratified human epidermal keratinocytes, complete with stratum corneum, making it relevant to test the interactions of microorganisms residing topically or that have penetrated the skin barrier. In this report we investigated the effects of topical and subcutaneous S. epidermidis, S. aureus and P. acnes, on the elicitation of keratinocyte inflammatory responses and the differentiation and proliferation of the RHE keratinocytes. We demonstrate the effects of speciesspecific responses and discuss how epidermal keratinocytes are impacted by and respond to the skin microflora as resident inhabitants of the skin surface and as opportunistic pathogens when the barrier is breached.

\section{Results}

Effects of bacteria added in the medium (breached barrier model) on keratinocyte inflammation and viability We tested the effects of bacteria added to the RHE medium, essentially underneath the RHE keratinocytes in order to mimic a scenario in which the bacteria had penetrated the epidermal barrier. Following treatment, the RHE culture medium was screened for pro-inflammatory cytokines and LDH as previously described. All of the tested species induced significant increases in the release of pro-inflammatory cytokines from the RHE keratinocytes and LDH (Figures 1, 2), indicating a pro-inflammatory and cytotoxic effect of the bacteria upon breaching of the epidermal barrier. We observed differential inflammatory and cytotoxic potency between species: P. acnes for example was least potent, inducing statistically significant increases in pro-inflammatory cytokine and LDH release only at the highest concentration tested. S. epidermidis had a greater pro-inflammatory and cytotoxic effect upon the RHE keratinocytes than $P$. acnes, inducing greater relative increases in cytokine and LDH release at each tested concentration, and induced statistically significant increases in cytokine and LDH release at lower concentrations than P. acnes (Figure 1). S. aureus was the most cytotoxic and pro-inflammatory of the species added to the RHE medium and stimulated a large and statistically significant increase in IL- $1 \alpha$ and LDH release when added at just $1 \times 10^{7} \mathrm{CFU}$ (Figure 2). Higher concentrations of $S$. aureus did not elicit greater increases in $\mathrm{LDH}$, suggesting that maximal RHE keratinocyte cell death had been induced. S. aureus was even able to stimulate statistically significant increases in IL- $1 \alpha$ and LDH release when added to the RHE medium at $1 \times 10^{2} \mathrm{CFU}$ (data not shown), showing that it is highly cytotoxic. All RHEs treated with $S$. aureus detached from their basement filters, indicating total keratinocyte cell death. It is likely that the cytotoxicity of $S$. aureus in the medium induced cell death, causing the release of stored IL- $1 \alpha$ before synthesis of IL- 8 and TNF $\alpha$ could occur and the levels of IL- 8 and TNF $\alpha$ released remained unaltered by $S$. aureus in the medium.

\section{Effects of bacteria added in the medium (breached barrier} model) on keratinocyte differentiation and proliferation status We studied the effects of bacteria added to the RHE medium on the keratinocyte expression of the genetic 


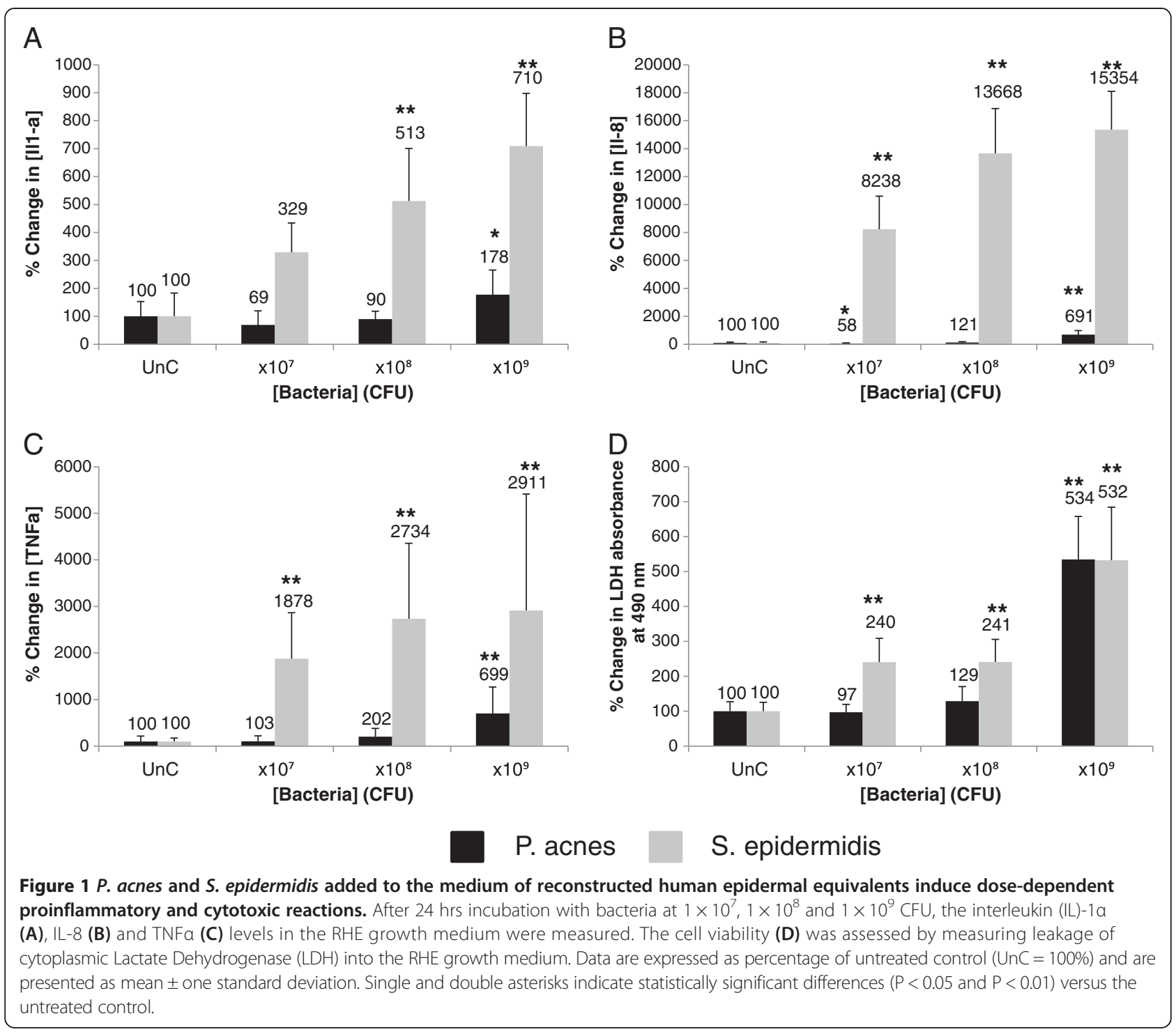

differentiation and proliferation markers: FLG, TG1, OCCL, CLD and PCNA. Addition of S. aureus in the medium induced keratinocyte cell death and degradation of marker mRNA, making it impossible to collect useful data. However, addition of $P$. acnes and S. epidermidis induced common patterns of changes in gene expression indicating a conserved response of the keratinocytes (Figure 3). In both cases, adding the bacteria to the medium induced decreased FLG and PCNA expression and increased OCCL and TG1 expression. The expression of CLD was not significantly affected by the presence of $S$. epidermidis or $P$. acnes in the RHE growth medium. As observed with the release of proinflammatory cytokines, S. epidermidis induced a stronger response than $P$. acnes in the induction of transcriptional changes of the RHE keratinocytes. S. epidermidis was able to induce significant changes in the expression of FLG, TG1, OCCL and PCNA when tested at only $1 \times 10^{7} \mathrm{CFU}$, whereas $P$. acnes was only able to induce significant increases in the affected differentiation markers at $1 \times 10^{9} \mathrm{CFU}$ and PCNA at $1 \times 10^{8} \mathrm{CFU}$.

Effects of topically applied bacteria (intact barrier model) on keratinocyte inflammation and viability

We then tested the potential of topically applied S. epidermidis, $P$. acnes, and $S$. aureus to induce proinflammatory cytokine release and cell death in human epidermal keratinocytes. Twenty four hours after treatment of RHEs with topically applied bacteria, RHE culture medium was taken and levels of the proinflammatory cytokines IL- $1 \alpha$, IL- 6 , IL-8, and TNF $\alpha$, as well as LDH were quantified to measure epidermal inflammation and cell death respectively. We observed that $P$. acnes and S. epidermidis had no 


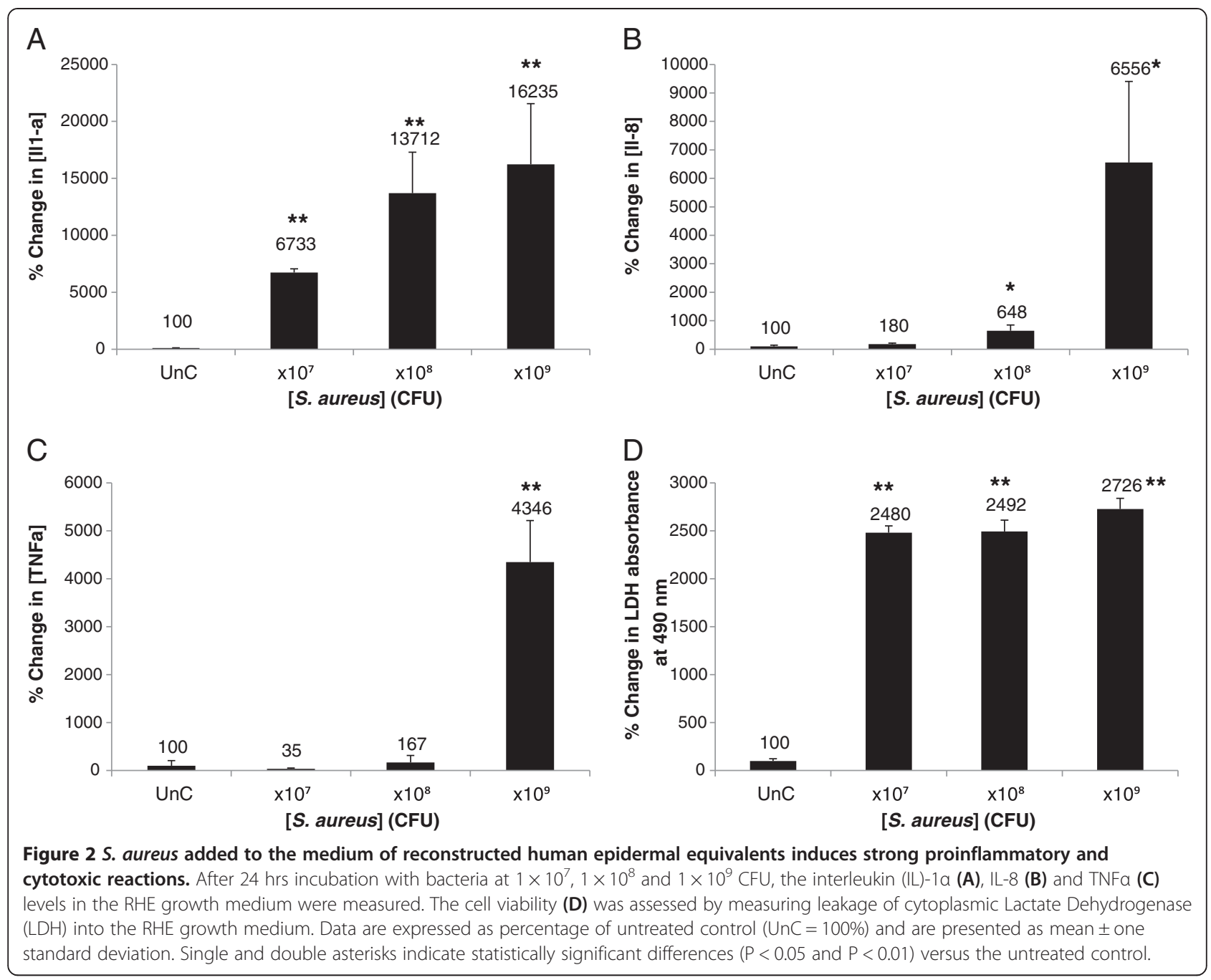

impact on the inflammatory status of human epidermal keratinocytes when applied topically and induced little change in IL-1 $\alpha$, IL-8 and TNF $\alpha$ release (Table 1). Topical S. epidermidis at $1 \times 10^{9} \mathrm{CFU}$ induced a statistically significant increase in IL-8 release compared to the untreated series $(P=0.05)$. However, the magnitude of this increase could be considered negligible and was elicited only at high bacterial concentrations. Neither species appeared to have cytotoxic effects on the RHE keratinocytes, based on the levels of LDH recovered from the RHE culture medium. S. aureus stimulated release IL- $1 \alpha$ from the RHE keratinocytes when topically applied only at high concentrations $\left(1 \times 10^{8}\right.$ and $\left.1 \times 10^{9} \mathrm{CFU}\right)$. This was observed in some RHE replicates, but not others, accounting for the high variability observed in the data. Coinciding with this, we also observed large increases in LDH release stimulated in these same replicates. It is likely that topically applied $S$. aureus at $1 \times 10^{8}$ and $1 \times 10^{9} \mathrm{CFU}$ induced keratinocyte cell death and subsequent release of stored
IL-1 $\alpha$ [21], before synthesis of IL- 8 and TNF $\alpha$ could occur. No IL- 6 was detected in the growth medium of the untreated or treated series.

\section{Effects of topically applied bacteria (intact barrier model)} on keratinocyte differentiation and proliferation

Finally, we tested whether topically applied S. epidermidis, $P$. acnes and $S$. aureus could affect the differentiation and proliferation of RHE keratinocytes. S. epidermidis and $P$. acnes were tested at $1 \times 10^{7}, 1 \times 10^{8}$, and $1 \times 10^{9} \mathrm{CFU}$, while $S$. aureus was tested at $1 \times 10^{3}, 1 \times 10^{5}$, and $1 \times 10^{7} \mathrm{CFU}$ as its cytotoxicity at high concentrations caused degradation of mRNA prohibiting further genetic analysis. The keratinocyte expression of the differentiation markers FLG (Profilaggrin), TG1 (Transglutaminase-1), CLD (Claudin-1) and OCCL (Occludin), as well as the proliferation marker PCNA (Proliferating Cell Nuclear Antigen), were measured following topical treatment with the tested species. None of the tested species induced any marked or significant 

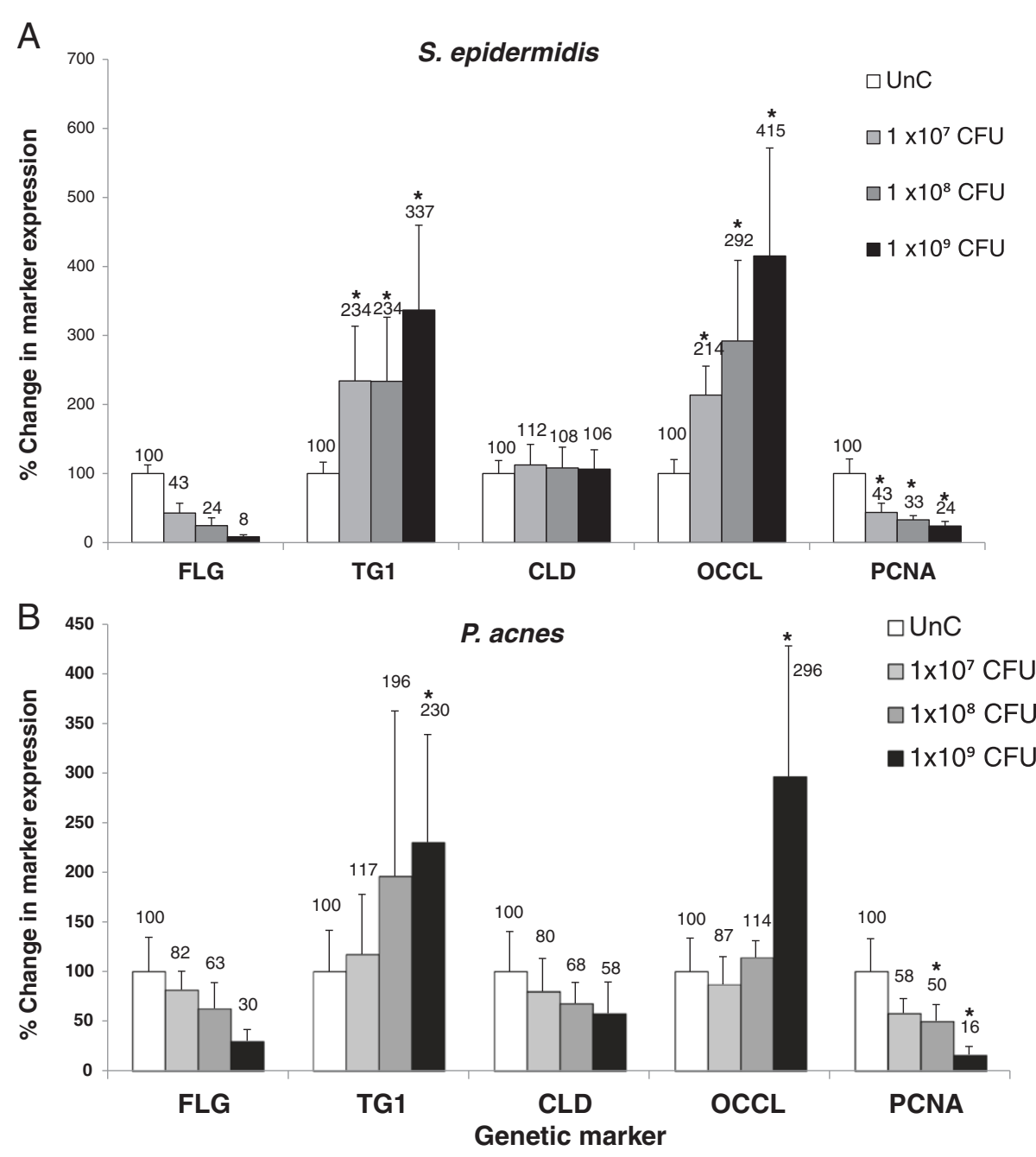

Figure 3 (A) S. epidermidis and (B) P. acnes added to the medium of reconstructed human epidermal equivalents induce dose-dependent changes in the expression of genes involved in differentiation (FLG, TG1, CLD, OCCL) and proliferation (PCNA). After 24 hrs incubation with bacteria at $1 \times 10^{7}, 1 \times 10^{8}$ and $1 \times 10^{9} \mathrm{CFU}$, RNA from the RHEs was extracted and reverse transcribed. The mRNA expression levels of a range of genes were determined by quantitative reverse transcription polymerase chain reaction. The amount of target transcripts was normalized using an $18 \mathrm{~S}$ RNA normalization gene. Data are expressed as percentage of untreated control $(\mathrm{UnC}=100 \%)$ and are presented as mean \pm one standard deviation. Asterisks indicate statistically significant differences $(P<0.05)$ vs the untreated control.

change in the expression of the differentiation or proliferation markers at the tested concentrations (Table 2).

\section{Discussion}

In this study we investigated whether the physical barrier presented by the stratum corneum is sufficient to inhibit microbial-induced effects on epidermal inflammation, differentiation, and proliferation status. Three bacterial species commonly found on the human skin surface $(P$. acnes, $S$. epidermidis and $S$. aureus) were tested for their effects on human epidermal keratinocytes in a stratified in vitro model. We show that bacteria introduced into the RHE medium (below the epidermal barrier) induced important changes whereas bacteria applied topically had little effect on the RHE keratinocytes.

Topically applied P. acnes and S. epidermidis did not elicit significant inflammatory responses from the RHE keratinocytes and displayed no cytotoxicity. Both $P$. acnes and $S$. epidermidis can be considered on one hand as commensal species, while on the other as opportunistic pathogens implicated in inflammatory diseases. Our data suggest that $P$. acnes and $S$. epidermidis are unlikely to contribute to the pathogenesis of inflammatory conditions when residing on top of the skin barrier: especially as these species were tested at concentrations orders of magnitude above the concentrations of between 3 and 6 CFU normally expected on healthy skin [22]. S. aureus however did 
Table 1 Average release of the proinflammatory cytokines IL-1a, IL-8, TNFa (pg/ml) and LDH (measured by optical absorbance at $490 \mathrm{~nm}$ ) from RHE 24 hours after topical treatment with S. epidermidis, P. acnes and S. aureus at different concentrations

\begin{tabular}{|c|c|c|c|c|}
\hline & Untreated control & $1 \times 10^{7} \mathrm{CFU}$ & $1 \times 10^{8} \mathrm{CFU}$ & $1 \times 10^{9} \mathrm{CFU}$ \\
\hline \multicolumn{5}{|l|}{ S. epidermidis } \\
\hline [IL-1a] released & $100 \pm 3.5$ & $116 \pm 111.2$ & $143.6 \pm 156.8$ & $186.8 \pm 215.8$ \\
\hline [IL-8] released & $100 \pm 18.4$ & $151.7 \pm 248.8$ & $118.9 \pm 156$ & $193.4 \pm 239.5^{*}$ \\
\hline [TNFa] released & $100 \pm 0.6$ & $124.4 \pm 35.6$ & $138 \pm 77.1$ & $151.8 \pm 80.7$ \\
\hline LDH absorbance & $100 \pm 0.03$ & $83.6 \pm 63.2$ & $82.6 \pm 62.9$ & $88.6 \pm 58.2$ \\
\hline \multicolumn{5}{|l|}{ P. acnes } \\
\hline$[\| \mathrm{L}-1 \mathrm{a}]$ released & $100 \pm 8.28$ & $123.1 \pm 100.3$ & $122 \pm 91$ & $133.4 \pm 112.1$ \\
\hline [IL-8] released & $100 \pm 42.08$ & $120.7 \pm 129.6$ & $109.5 \pm 97.3$ & $107.1 \pm 84.9$ \\
\hline [TNFa] released & $100 \pm 1.6$ & $162.6 \pm 158.1$ & $115.7 \pm 126.1$ & $88.6 \pm 113.6$ \\
\hline LDH absorbance & $100 \pm 0.04$ & $100.2 \pm 148.4$ & $108.8 \pm 161.6$ & $102.1 \pm 142.8$ \\
\hline \multicolumn{5}{|l|}{ S. aureus } \\
\hline$[\| \mathrm{L}-1 \mathrm{a}]$ released & $100 \pm 2.3$ & $157.6 \pm 303.8$ & $1062.1 \pm 3556.2$ & $1251.7 \pm 3735.6$ \\
\hline [IL-8] released & $100 \pm 12.1$ & $60.4 \pm 27.7$ & $93.1 \pm 95.1$ & $73 \pm 2.6$ \\
\hline LDH absorbance & $100 \pm 0.1$ & $273.3 \pm 357.5$ & $505.2 \pm 1002$ & $666.4 \pm 1321$ \\
\hline
\end{tabular}

Results are expressed as percentage of the untreated control.

*Single asterisk indicate statistically significant differences $(P<0.05)$ versus the untreated control.

Table 2 Average expression of the RHE differentiation markers FLG, TG1, CLD, OCCL and the proliferation marker PCNA (measured as \% expression of untreated control) 24 hours after topical treatment with $S$. epidermidis, $P$. acnes and $S$. aureus at different concentrations

\begin{tabular}{|c|c|c|c|c|}
\hline & $\begin{array}{l}\text { Untreated } \\
\text { control }\end{array}$ & $1 \times 10^{7} \mathrm{CFU}$ & $1 \times 10^{8} \mathrm{CFU}$ & $1 \times 10^{9} \mathrm{CFU}$ \\
\hline \multicolumn{5}{|c|}{ S. epidermidis } \\
\hline FLG & $100 \pm 21.1$ & $105 \pm 50.5$ & $113 \pm 60$ & $99 \pm 37.4$ \\
\hline TG1 & $100 \pm 11$ & $95 \pm 30.7$ & $95 \pm 33.5$ & $92 \pm 25.8$ \\
\hline CLD & $100 \pm 29.2$ & $142 \pm 68.3$ & $161 \pm 105.2^{*}$ & $123 \pm 53.5$ \\
\hline $\mathrm{OCCL}$ & $100 \pm 17.2$ & $84 \pm 30.9$ & $95 \pm 35.4$ & $113 \pm 63.8$ \\
\hline PCNA & $100 \pm 25.3$ & $129 \pm 36.2$ & $120 \pm 39.2$ & $135 \pm 34^{*}$ \\
\hline \multicolumn{5}{|l|}{ P. acnes } \\
\hline FLG & $100 \pm 28$ & $110 \pm 26.5$ & $101 \pm 42$ & $99 \pm 31.4$ \\
\hline TG1 & $100 \pm 26.9$ & $125 \pm 74.7$ & $84 \pm 29.9$ & $77 \pm 34.6$ \\
\hline CLD & $100 \pm 36.5$ & $90 \pm 35.7$ & $71 \pm 32.1^{*}$ & $84 \pm 31.6$ \\
\hline $\mathrm{OCCL}$ & $100 \pm 33.4$ & $112 \pm 72.9$ & $81 \pm 40.5$ & $97 \pm 42.6$ \\
\hline PCNA & $100 \pm 18.6$ & $102 \pm 49.4$ & $132 \pm 108.2$ & $140 \pm 93.9$ \\
\hline S. aureus & & $1 \times 10^{3} \mathrm{CFU}$ & $1 \times 10^{5} \mathrm{CFU}$ & $1 \times 10^{7} \mathrm{CFU}$ \\
\hline FLG & $100 \pm 19.3$ & $121 \pm 32.6$ & $107 \pm 20.5$ & $141 \pm 45.9$ \\
\hline TG1 & $100 \pm 12$ & $179 \pm 65.8$ & $112 \pm 31.7$ & $114 \pm 34.3$ \\
\hline CLD & $100 \pm 22.4$ & $107 \pm 25.3$ & $126 \pm 30.2$ & $141 \pm 43.8^{*}$ \\
\hline $\mathrm{OCCL}$ & $100 \pm 31.9$ & $139 \pm 67.7$ & $141 \pm 43.9$ & $143 \pm 45.7^{*}$ \\
\hline PCNA & $100 \pm 18$ & $104 \pm 22.6$ & $98 \pm 14.3$ & $97 \pm 48$ \\
\hline
\end{tabular}

*Single asterisk indicate statistically significant differences $(P<0.05)$ versus the untreated control. display limited proinflammatory and cytotoxic effects in some replicates when tested at high concentrations. However, it is unlikely that concentrations of $S$. aureus would reach these values on top of human skin in vivo, unless the bacteria could overpower a weakened immune system. We also note that none of the tested species were able to alter keratinocyte differentiation or proliferation and our data suggest that topically residing skin bacteria cannot influence the development of the human skin. The barrier posed by the stratum corneum of the RHE model is likely to have prevented the contact and interaction of the tested bacteria with the living RHE keratinocytes, and may prevent the topical skin microflora affecting living keratinocytes in vivo. S. aureus however, has been shown to be able to circumvent the stratum corneum barrier in the pathogenesis of diseases such as scalded skin syndrome [23], which may account for the inflammatory effects it induced when topically applied to the RHE models.

When added to the RHE growth medium, the tested species had a large impact on the RHE keratinocytes. $P$. acnes and S. epidermidis were able to induce large inflammatory responses from the RHE keratinocytes and were highly cytotoxic. These effects were stronger when induced by $S$. epidermidis. P. acnes and S. epidermidis are known to cause infection when introduced to the subcutaneous space by prostheses or catheters that penetrate the skin [17]. In these situations, the concentrations of $P$. acnes or $S$. epidermidis underneath the skin barrier could equal those tested in this experiment and cause large-scale 
inflammation and tissue death in vivo. S. aureus induced complete keratinocyte cell death and subsequent IL-1 $\alpha$ release even when tested at low concentrations, indicating the highly pathogenic potential of $S$. aureus upon penetration of the skin barrier.

Specific transcriptional changes in keratinocyte marker expression were also induced when the skin bacteria were added to the RHE culture medium. P. acnes and S. epidermidis induced identical expression profiles in the keratinocyte markers that appeared to indicate an effort to repair the epidermal barrier function. We observed large increases in the expression of TG1 in RHE keratinocytes treated with $P$. acnes and $S$. epidermidis in the medium. Transglutaminase-mediated crosslinking of protein scaffold networks is an important step in epidermal keratinocyte terminal differentiation, which results in the formation of the stratum corneum barrier (reviewed by Eckert et al. [24]) and the upregulation of TG1 suggests an increased effort to improve epidermal barrier function by the keratinocytes. It is therefore surprising that expression of FLG was downregulated by the bacterial treatment: FLG monomer binding to keratin filaments organizes them into bundles, establishing a protein network that forms the cornified envelope and stratum corneum [25]. Recently however, de Koning et al. [26] observed identical transcriptional changes in FLG and TG1 during epidermal barrier repair, corroborating the hypothesis that $P$. acnes and S. epidermidis stimulated barrier repair processes in RHE keratinocytes when added to the medium. de Koning et al. [26] speculate that FLG may be involved in epidermal barrier maintenance, as opposed to repair and is subsequently downregulated as focus is shifted to repair. The tight junction protein OCCL was significantly upregulated by the bacterial treatments, which may indicate an effort of the keratinocytes to improve barrier function through tightening of cell-to-cell contacts. However, we did not observe altered expression of the tight junction protein CLD. It may instead be that OCCL is a degradation target for these bacteria and is upregulated in expression to compensate: OCCL has been shown to be degraded during staphylococcal infection previously [27]. We also observed large decreases in the expression of the proliferation marker, PCNA. One might expect the proliferation rate of RHE keratinocytes treated with bacteria to increase in order to mount an amplified immune response. However, it seems that the RHE keratinocytes favored differentiation and barrier repair over regeneration and proliferation. The specific transcriptional changes induced by bacteria introduced below the RHE epidermal barrier were identical between the tested species and matched previously described profiles elicited in response to barrier disruption [26]. It is possible that epidermal keratinocytes recognize the presence of the bacteria as a barrier disruption signal, as opposed to a scenario in which the microflora are able to modulate gene transcription in epidermal keratinocytes.

In this work we modeled epidermal breach by introducing bacteria to the medium of RHE. This is a first approach to understand skin barrier breach. A more realistic approach could be by damaging the barrier e.g. by scratching or tape stripping. These methods would be valuable for an in vivo setting and cannot be applicable and reproducible for the in vitro RHE model. However, the number of measured parameters in vivo would be limited, unless an invasive biopsy would be used with the associated problems (e.g. inflammatory markers will increase due to the invasive nature of the biopsy).

Another potential limitation of the study is that we used ATCC strains that could likely have distanced genetically from actual human skin bacteria. Isolation of microbial strains collected from human skin is going to be tried in the future on this model. However, genetic variability of such strains between hosts or even between body sites of the same host needs also to be considered.

Our data suggest that topically residing bacteria must be able to penetrate the epidermal barrier in order to affect the activities of epidermal keratinocytes. The human skin microflora has been shown to offer protective immunological benefits against pathogens both directly [4] as well as indirectly by interacting with human skin keratinocytes $[5,28]$. One scenario of this could be seen during wounding in which $S$. epidermidis is granted access to epidermal keratinocytes and can modulate their immune response [28]. However it is hard to envisage the topical skin microflora having these effects if prevented from interacting with the epidermal keratinocytes by the stratum corneum. We mentioned above the possibility that the skin microflora may provide epitopes to prime the immune system against pathogens following birth [6-8] and the possibility that this early colonization could stimulate the maturation of the skin barrier following birth. It is possible that the skin microflora may be able to interact with epidermal keratinocytes during this period and enter the epidermis via hair follicles and other epidermal appendages [7]. The diverse ways by which topically residing commensals may be able to interact with epidermal keratinocytes remain to be explored.

\section{Conclusions}

Based on the observed data, P. acnes, S. epidermidis and $S$. aureus were unable to modulate the inflammatory, differentiation and proliferation status of RHE keratinocytes when applied topically. When tested on a breached skin barrier model, $P$. acnes and S. epidermidis induced strong inflammatory responses from RHE keratinocytes and induced specific changes in the expression of RHE keratinocyte differentiation and proliferation markers, perhaps indicative of a barrier repair response. S. aureus 
induced complete keratinocyte cell death and release of proinflammatory cytokines when tested even at low concentrations. Together, our data show that these species of common skin bacteria are prevented from affecting human epidermal keratinocytes by the stratum corneum when residing topically, but may contribute to the pathogenesis of inflammatory diseases following penetration of the skin immunity barrier. The skin barrier is therefore an important part of the innate immune system's defence against the pathogenic effects of common skin bacteria.

\section{Methods}

\section{Preparation of bacteria}

P. acnes (ATCC strain 6919) from second passage stock at $-80^{\circ} \mathrm{C}$ was plated on tryptic soy agar for 5 days at $37^{\circ} \mathrm{C}$ in anaerobic conditions to yield third passage bacteria and was repeated to yield fourth passage bacteria. S. epidermidis (ATCC strain 12228) and S. aureus (ATCC strain 6538) were taken from second passage stock at $-80^{\circ} \mathrm{C}$ and were plated on tryptic soy agar for $48 \mathrm{hrs}$ at $32^{\circ} \mathrm{C}$ in aerobic conditions to yield third passage bacteria, and again to yield fourth passage bacteria. Each species of bacteria was suspended in both physiological water and RHE growth medium at $1 \times 10^{9}$ Colony Forming Units (CFU), as estimated by the optical density of the solutions, and confirmed by standard plate count.

\section{Reconstructed human epidermis (RHE)}

RHE tissues were purchased from Skinethic, Lyon, France. Upon delivery, RHE inserts were transferred to $1 \mathrm{ml}$ RHE growth medium free of antibiotics and hydrocortisone. Inserts were incubated for $24 \mathrm{hrs}$ at $37^{\circ} \mathrm{C}$ and $5 \% \mathrm{CO}_{2}$ in sterile conditions.

For the subcutaneous treatment, RHE inserts were transferred to wells containing $1 \mathrm{ml}$ RHE growth medium containing $P$. acnes, $S$. epidermidis or S. aureus at $1 \times 10^{7} \mathrm{CFU}$,
$1 \times 10^{8} \mathrm{CFU}$ and $1 \times 10^{9} \mathrm{CFU}$. For the untreated control, RHE inserts were transferred to $1 \mathrm{ml}$ RHE growth medium without bacteria.

For the topical treatment, $20 \mu \mathrm{l}$ of bacterial solution containing $1 \times 10^{7} \mathrm{CFU}, 1 \times 10^{8} \mathrm{CFU}$ and $1 \times 10^{9} \mathrm{CFU}$ of $P$. acnes, S. epidermidis or S. aureus in physiological solution was added directly on top of RHE inserts. For the untreated control, $20 \mu \mathrm{l}$ of physiological solution without bacteria was applied instead. The solution applied as a drop was then spread uniformly on the surface with a sealed end Pasteur pipette.

For $S$ aureus, lower concentrations $\left(1 \times 10^{2}, 1 \times 10^{3}\right.$, $1 \times 10^{5}$ and $1 \times 10^{7} \mathrm{CFU}$ ) were also tested in the medium as this species induces cell death at high concentrations.

All treated RHEs were incubated for $24 \mathrm{hrs}$ at $37^{\circ} \mathrm{C}$ and $5 \% \mathrm{CO}_{2}$ before the RHE medium was collected and frozen at $-80^{\circ} \mathrm{C}$ and RNA was extracted. The represented values are the mean and the standard deviation of at least three to five independent experiments.

\section{Biochemical assays}

Collected RHE growth medium was screened for Interleukin (IL)-1 $\alpha$, IL-6, IL-8 and Tumor Necrosis Factor- $\alpha$ (TNF $\alpha$ ) using the Flourokine MAP Human Base Kit A (R\&D Systems, Lille, France) and analyzed on the Bioplex200 (Bio-rad, Marnes-la-Coquette, France).

Keratinocyte cell viability was estimated by measuring leakage of cytoplasmic Lactate Dehydrogenase (LDH) into the RHE growth medium using the TOX7-1KT Lactate Dehydrogenase-based In-Vitro Toxicology Assay Kit (Sigma-Aldrich, Saint Quentin Fallavier, France) with the procedure provided by the manufacturer.

Results were expressed as percentage change in inflammation markers or in LDH absorbance at $490 \mathrm{~nm}$, compared to the untreated control.

Table 3 Sense and antisense primer sequences used in qPCR

\begin{tabular}{|c|c|c|c|}
\hline Genetic marker & & Primer & Sequence \\
\hline \multirow[t]{2}{*}{ 18S Ribosomal RNA gene } & & Forward & 5'-TAAGTCCCTGCCCTITGTACACA-3' \\
\hline & & Reverse & 5'-GATCCGAGGGCCTCACTAAAC-3' \\
\hline \multirow[t]{2}{*}{ Profilaggrin } & FLG & Forward & 5'-TGGCAAATCATCATCTCAAGTG-3' \\
\hline & & Reverse & 5'-CTGTCCTGGCTAACTCTGG-3' \\
\hline \multirow[t]{2}{*}{ Occludin } & $\mathrm{OCCL}$ & Forward & 5'-CAAGCGGTIITATCCAGAGTC-3' \\
\hline & & Reverse & 5'-AAGTCATCCACAGGCGAAG-3' \\
\hline \multirow[t]{2}{*}{ Claudin1 } & CLD1 & Forward & 5'-AGTGCTTGGAAGACGATGAG-3' \\
\hline & & Reverse & 5'-TTGAACGATTCTATTGCCATACC-3' \\
\hline \multirow[t]{2}{*}{ Transglutaminase 1} & TG1 & Forward & 5'-TGCTGGATGCCTGCTTATAC-3' \\
\hline & & Reverse & 5'-TGCCTCGGGAGTAATCACC-3' \\
\hline \multirow[t]{2}{*}{ Proliferating cell nuclear antigen } & PCNA & Forward & 5'-AACCTCACCAGTATGTCC-3' \\
\hline & & Reverse & 5'-ATCCATCAACTTCATTTCATAG-3' \\
\hline
\end{tabular}




\section{Measuring changes in keratinocyte gene expression}

Each RHE epidermal tissue was physically separated from the inert polycarbonate filter and lysed in $400 \mu \mathrm{l}$ lysis buffer, consisting of 100 parts RLT buffer (from the Fibrous Tissue Mini Kit (50) (Qiagen, Courtaboeuf, France), to one part 2-Mercaptoethanol (Sigma-Aldrich) inside a ceramic bead tube (Ozyme, Saint Quentin en Yvelines, France). The tubes were shaken at $5000 \mathrm{rpm}$ and RNA was extracted from the solutions using the Fibrous Tissue Mini Kit (50) on the automated RNA extraction unit; the Qiacube (Qiagen) which eluted the samples in $60 \mu \mathrm{l}$ RLT buffer.

Reverse transcription was performed with the High Capacity Reverse Trancription Kit (Applied Biosystems, Courtaboeuf, France) using $2 \mu \mathrm{l}$ RT Buffer, $0.8 \mu \mathrm{l}$ dNTP mix, $2 \mu \mathrm{l} 10 \mathrm{x}$ RT random primers and $4.2 \mu \mathrm{l}$ nuclease free water per $10 \mu \mathrm{l}$ sample. The RT reaction was performed at $25^{\circ} \mathrm{C}$ for $10 \min 37^{\circ} \mathrm{C}$ for $2 \mathrm{hrs}$.

Quantitative Polymerase Chain Reaction (qPCR) was used to screen for the expression of genes filaggrin (FLG), transglutaminase 1 (TG1), occludin (OCCL), claudin (CLD), and Proliferating Cell Nuclear Antigen (PCNA). The specific primers used in the study are shown in Table 3. The expression of these genes was normalized against the expression of the $18 \mathrm{~S}$ housekeeping gene. For each PCR reaction, $5 \mu \mathrm{l}$ DNA solution was added to $12.5 \mu \mathrm{l}$ Power SYBR Green PCR Master Mix (Applied Biosystems), $0.075 \mu \mathrm{l} \mathrm{L}$-primer solution, $0.075 \mu \mathrm{l}$ R-primer solution (Sigma-Aldrich) and $7.35 \mu \mathrm{l}$ nuclease free water. The reactions were cycled in a 96-well PCR plate on the Mx3000p Stratagene, (Agilent, Massy, France). Changes in expression of a genetic marker were deemed significant if found to be half or double that of the untreated control.

\section{Statistical analysis}

Comparison of keratinocyte Interleukin release under test conditions versus untreated control were performed using the Student's $t$-test at 95\% significance level.

\section{Abbreviations \\ CFU: Colony forming units; CLD: Claudin; dNTP: Deoxyribonucleotide phosphate; FLG: Filaggrin; IL-1a: Interleukin 1-alpha; IL-6: Interleukin-6; IL-8: Interleukin-8; LDH: Lactose dehydrogenase; NHEK: Normal human epidermal keratinocyte; OCCL: Occludin; PCNA: Proliferating cell nuclear antigen; qPCR: Quantitative polymerase chain reaction; RHE: Reconstructed human epidermis; RT: Reverse transcriptase; TG1: Transglutaminase-1; TLR: Toll-like-receptor; TNFa: Tumour necrosis factor alpha.}

\section{Competing interest}

The authors are employees of Johnson \& Johnson Santé Beauté France.

\section{Authors' contributions}

The experiments were designed by GS and TO and were carried out by PD, with the exception of the preparation of bacterial solutions which was performed by DY. The project was supervised by JS and HKW. PD prepared the final manuscript, which was checked and approved by all authors.

\section{Acknowledgements}

The authors would like to thank Christelle Fécourt for her technical assistance with the GPCR experiment. This study was fully funded by Johnson \& Johnson Santé Beauté France.

\section{Author details}

${ }^{1}$ Durham University, School of Biological and Biomedical Sciences, South

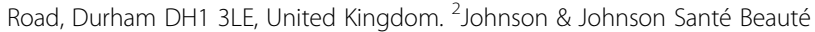
France, Campus de Maigremont, Val de Reuil 27100, France. ${ }^{3}$ Johnson \& Johnson Santé Beauté France, 1 rue Camille Desmoulins, Issy-les-Moulineaux 92787, France.

Received: 24 April 2013 Accepted: 12 November 2013 Published: 18 November 2013

\section{References}

1. Wanke I, Steffen H, Christ C, Krismer B, Gotz F, Peschel A, Schaller M, Schittek B: Skin commensals amplify the innate immune response to pathogens by activation of distinct signaling pathways. $J$ Invest Dermatol 2011, 131:382-390.

2. Grange PA, Chereau C, Raingeaud J, Nicco C, Weill B, Dupin N, Batteux F: Production of superoxide anions by keratinocytes initiates $P$. acnes-induced inflammation of the skin. PLOS Pathog 2009, 5:e1000527.

3. Pivarcsi A, Nagy I, Kemeny L: Innate immunity in the skin: how keratinocytes fight against pathogens. Curr Immunol Rev 2005, 1:29-42.

4. Cogen AL, Yamasaki K, Sanchez KM, Dorschner RA, Lai Y, MacLeod DT, Torpey JW, Otto M, Nizet V, Kim JE, Gallo RL: Selective antimicrobial action is provided by phenol-soluble modulins derived from Staphylococcus epidermidis, a normal resident of the skin. J Invest Dermatol 2010, 130:192-200.

5. Lai Y, Cogen AL, Radek KA, Park HJ, Macleod DT, Leichtle A, Ryan AF, Di Nardo A, Gallo RL: Activation of TLR2 by a small molecule produced by Staphylococcus epidermidis increases antimicrobial defense against bacterial skin infections. J Invest Dermatol 2010, 130:2211-2221.

6. Capone KA, Dowd SE, Stamatas GN, Nikolovski J: Diversity of the human skin microbiome early in life. J Invest Dermatol 2011, 131:2026-2032.

7. Marchini G, Nelson A, Edner J, Lonne-Rahm S, Stavreus-Evers A, Hultenby K: Erythema toxicum neonatorum is an innate immune response to commensal microbes penetrated into the skin of the newborn infant. Pediatr Res 2005, 58:613-616.

8. Naik S, Bouladoux N, Wilhelm C, Molloy MJ, Salcedo R, Kastenmuller W, Deming C, Quinones M, Koo L, Conlan S, et al: Compartmentalized control of skin immunity by resident commensals. Science 2012, 337:1115-1119.

9. Grice EA, Kong HH, Conlan S, Deming CB, Davis J, Young AC, Bouffard GG, Blakesley RW, Murray PR, Green ED, et al: Topographical and temporal diversity of the human skin microbiome. Science 2009, 324:1190-1192.

10. Henderson CA, Taylor J, Cunliffe WJ: Sebum excretion rates in mothers and neonates. Br J Dermatol 2000, 142:110-111.

11. Nikolovski J, Stamatas GN, Kollias N, Wiegand BC: Barrier function and water-holding and transport properties of infant stratum corneum are different from adult and continue to develop through the first year of life. J Invest Dermatol 2008, 128:1728-1736.

12. Hoeger PH, Enzmann CC: Skin physiology of the neonate and young infant: a prospective study of functional skin parameters during early infancy. Pediatr Dermatol 2002, 19:256-262.

13. Charles AJ: Superficial cutaneous fungal infections in tropical countries. Dermatol Ther 2009, 22:550-559.

14. Bruggemann $\mathrm{H}$ : Insights in the pathogenic potential of Propionibacterium acnes from its complete genome. Semin Cutan Med Surg 2005, 24:67-72.

15. Kong HH: Skin microbiome: genomics-based insights into the diversity and role of skin microbes. Trends Mol Med 2011, 17:320-328.

16. Balci DD, Duran N, Ozer B, Gunesacar R, Onlen Y, Yenin JZ: High prevalence of Staphylococcus aureus cultivation and superantigen production in patients with psoriasis. Eur J Dermatol 2009, 19:238-242.

17. Cogen AL, Nizet V, Gallo RL: Skin microbiota: a source of disease or defence? Br J Dermatol 2008, 158:442-455.

18. Stamatas GN, Nikolovski J, Luedtke MA, Kollias N, Wiegand BC: Infant skin microstructure assessed in vivo differs from adult skin in organization and at the cellular level. Pediatr Dermatol 2010, 27:125-131. 
19. Jarrousse V, Castex-Rizzi N, Khammari A, Charveron M, Dreno B: Modulation of integrins and filaggrin expression by Propionibacterium acnes extracts on keratinocytes. Arch Dermatol Res 2007, 299:441-447.

20. Akaza N, Akamatsu H, Kishi M, Mizutani H, Ishii I, Nakata S, Matsunaga K: Effects of Propionibacterium acnes on various mRNA expression levels in normal human epidermal keratinocytes in vitro. J Dermatol 2009, 36:213-223.

21. Coquette A, Berna N, Vandenbosch A, Rosdy M, De Wever B, Poumay Y: Analysis of interleukin-1alpha (IL-1alpha) and interleukin-8 (IL-8) expression and release in in vitro reconstructed human epidermis for the prediction of in vivo skin irritation and/or sensitization. Toxicol In Vitro 2003, 17:311-321.

22. Reichel M, Heisig P, Kampf G: Identification of variables for aerobic bacterial density at clinically relevant skin sites. J Hosp Infect 2011, 78:5-10.

23. Hanakawa Y, Schechter NM, Lin C, Garza L, Li H, Yamaguchi T, Fudaba Y, Nishifuji K, Sugai M, Amagai M, Stanley JR: Molecular mechanisms of blister formation in bullous impetigo and staphylococcal scalded skin syndrome. J Clin Invest 2002, 110:53-60.

24. Eckert RL, Sturniolo MT, Broome AM, Ruse M, Rorke EA: Transglutaminase function in epidermis. J Invest Dermatol 2005, 124:481-492.

25. Sandilands A, Sutherland C, Irvine AD, McLean WH: Filaggrin in the frontline: role in skin barrier function and disease. J Cell Sci 2009, 122:1285-1294.

26. de Koning HD, van den Bogaard EH, Bergboer JG, Kamsteeg M, van Vlijmen-Willems IM, Hitomi K, Henry J, Simon M, Takashita N, Ishida-Yamamoto A, et al: Expression profile of cornified envelope structural proteins and keratinocyte differentiation-regulating proteins during skin barrier repair. Br J Dermatol 2012, 166:1245-1254.

27. Ohnemus U, Kohrmeyer K, Houdek P, Rohde H, Wladykowski E, Vidal S, Horstkotte MA, Aepfelbacher M, Kirschner N, Behne MJ, et al: Regulation of epidermal tight-junctions (TJ) during infection with exfoliative toxin-negative Staphylococcus strains. J Invest Dermatol 2008, 128:906-916.

28. Lai Y, Di Nardo A, Nakatsuji T, Leichtle A, Yang Y, Cogen AL, Wu ZR, Hooper LV, Schmidt RR, von Aulock S, et al: Commensal bacteria regulate toll-like receptor 3-dependent inflammation after skin injury. Nat Med 2009, 15:1377-1382.

doi:10.1186/1756-0500-6-474

Cite this article as: Duckney et al:: The role of the skin barrier in modulating the effects of common skin microbial species on the inflammation, differentiation and proliferation status of epidermal keratinocytes. BMC Research Notes 2013 6:474.

\section{Submit your next manuscript to BioMed Central and take full advantage of:}

- Convenient online submission

- Thorough peer review

- No space constraints or color figure charges

- Immediate publication on acceptance

- Inclusion in PubMed, CAS, Scopus and Google Scholar

- Research which is freely available for redistribution 